\title{
Wanted: A Theoretical Roadmap to Research and Practice Across Individual, Interpersonal, and Structural Levels of Analysis
}

\author{
Dolores Albarracin • Alexander J. Rothman • \\ Ralph Di Clemente - Carlos del Rio
}

Published online: 8 September 2010

(C) Springer Science+Business Media, LLC 2010

Investigators routinely acknowledge the existence and relevance of the different systems that regulate behavior, yet their empirical work remains focused on processes that operate within a single system. In order for investigators to conduct basic and applied initiatives that cross levels of analysis, theoretical principles are needed that delimit the interplay between different systems. Thus, a systematic effort is needed to integrate behavior change principles across levels or systems. As shown in this supplement, the proposed integration is necessary, likely impactful, and timely, and derived from the work of researchers from basic and applied areas who agreed to undertake this initiative as part of expert teams who would interact in person during a 2-day conference that took place Atlanta, GA in 2009. A number of observations detailed below motivated the organization of the team work leading to this supplement of AIDS and Behavior.

There has been substantial theoretical integration about behavior change at the individual level. Considerable theoretical integration has taken place regarding the processes that underlie changes in individual beliefs, attitudes, and behaviors. In fact, the HIV epidemic of the 1980's stimulated the uniting of funds and expertise from various disciplines in the development of a shared behavior-change

D. Albarracin $(\bowtie)$

Department of Psychology, University of Illinois, 603 E. Daniel St, Champaign Urbana, IL 61820, USA

e-mail: dalbarra@ufl.edu

R. Di Clemente · C. del Rio

Rollins School of Public Health, Emory University, Atlanta, GA, USA

\section{A. J. Rothman}

Department of Psychology, University of Minnesota, Minneapolis, MN, USA paradigm. As a key example, in 1992, a group of behavioral researchers joined forces-upon request from the National Institute of Mental Health-to develop a paradigm for behavior change that would guide research and practice in the prevention of HIV [1]. Various models were examined and the key assumptions were condensed into a limited number of principles that clearly illuminated subsequent preventive research [2]. Parallel to that, various integrative models were proposed and tested [3], leading to the development of cutting-edge interventions to change risky behaviors (e.g., sex risk behavior, antiretroviral adherence) in many places in the world. Furthermore, some of the assumptions that underlie these integrations were formally tested in a large meta-analysis of the outcomes of HIV prevention research [2]. As a result, researchers and practitioners have a set of well-tested principles of behavior change they can use for HIV prevention at the individual level. Nonetheless, limitations in the magnitude of the effects of resulting interventions suggest that other levels must be brought in to increase preventive success.

Emerging HIV-prevention interventions must address other levels of analysis that are NOT currently incorporated in the existing theoretical integration. Despite the importance of past individual-level theoretical integrations, emerging interventions are attempting to modify behavioral and disease determinants that operate at interpersonal and structural levels (for the general concerns, see [4-6]; in the domain of HIV, [7-11]). These approaches are invaluable and point toward promising strategies that may increase the degree to which behavior can be modified. Up to now, however, the depth of theoretical integration targeted at the individual level has not been applied to these higher order levels of analysis. Thus, there are deficiencies in our current understanding of how intervening at the interpersonal or the community/social structures in which 
individuals live facilitates or inhibits individual belief change. Relevant questions that need to be examined include: What is the impact of interpersonal- and community-level interventions on the individual-level beliefs, emotions, and/or behaviors that can ultimately lead to change? What are the mediators and moderators of this impact? What are the reciprocal relations between changes in the individual and the broader systems? How can we explain these reciprocal relations? What techniques can produce change initiated at different levels? How can interventions at different levels be combined or designed to be complementary? What are the principles that guide such combinations? In sum, a critical goal of this supplement is to present frameworks that will afford the integration of theoretical principles across levels or systems that can guide the design and testing of innovative intervention strategies.

There are several indicators of the current absence of this theoretical integration. First, an examination of conference proceedings and two recent volumes edited by Ralph DiClemente $[12,13]$ confirmed that no such theoretical integration has yet been achieved in the area of HIV/ AIDS prevention. Perhaps more importantly, we conducted a literature search for "multi-level," "structural," "family," "policy," and "community interventions" in the domain of HIV prevention using the Science Citation Index. This review yielded 146 articles and shows a peak in production after the year 2000. Given that this is a new, emerging literature, it is not surprising that only one of the 146 articles was dedicated to some theoretical integration linking levels of analysis, a review by Ramirez-Valles [14]. He proposed that community involvement moderates the association between three socio-structural risk factors (i.e., poverty, homophobia, and racism) and sexual risk behavior. He also argued that community involvement in HIV/ AIDS organizations reduces sexual risk behavior by impacting peer norms, self-efficacy, positive self-identity, and alienation. Although this contribution suggests that the frameworks described in this supplement can be successful, the framework was not designed to address our proposed goal of a more comprehensive theoretical examination. First, it did not address all key determinants of behavior, focusing just on those traditionally linked to community influence (e.g., norms). Second, the family/couple level was not considered, nor where interactions or reciprocal relations among the different levels analyzed.

No integration of principles underlying behavior change has carefully considered clinical outcomes. The theoretical papers discussed previously have centered on behavior as the end point and have considered a limited set of preventive behaviors. As a result, a careful consideration of clinical outcomes that might result from intervening at various levels of analysis is essential to ensure efficacious interventions in the future. Another major limitation of the current literature is that behavioral theory has not been systematically applied to behaviors linked to recent clinical advances. For example, postexposure prophylaxis (PEP) is recommended for all exposure to HIV. Nonetheless, awareness of the method in the community is still low $[15,16]$, and there is no literature on the multi-level determinants of PEP-seeking behavior. HIV testing is another behavior that can be fruitfully analyzed from a multi-level perspective. One of the articles in this supplement discusses it extensively.

The theoretical integration must be undertaken by a community of basic and applied researchers with a broad range of experiences. We believe that generating an integrative theory about multi-determined change requires the close attention of a community of researchers whose research and practice emphasize different levels of analysis. The reason is that detailed knowledge of all these areas by a single researcher is practically impossible. However, it is clear that not any group of researchers can provide this integration. Therefore, we identified a specific set of objectives that would enable us to identify and recruit a group of researchers that is likely to succeed at this task.

1. The first objective was to recruit researchers with experience in individual-level theories. Researchers with experience in individual-level theories have identified key variables and addressed many possible interrelations among these variables. Moreover, to be effective, interventions at the interpersonal and community levels must impact the individual's behavior. As a result, researchers with individual-level modeling experience are well-poised to contribute to the theoretical integration between the individual and higherorder systems.

2. Another way of building the required community was to recruit outstanding researchers with experience working at the interpersonal and community/structural levels. Through the conference and conference preparation, these investigators were exposed to the theoretical integration that is available for individuallevel behavior. This exposure prepared them to connect their own knowledge about the interpersonal and community/structural levels to the principles from the individual level. Their knowledge and experience also shed light on the limitations of current individuallevel models and the many innovative individual models that are yet to be introduced to HIV-prevention researchers.

3. Another important principle in building the proposed theoretical integration was to recruit investigators who worked on diverse health issues. For example, the spectrum of research on interpersonal processes is 
broader than that currently being implemented in ongoing interpersonal interventions to promote physical health, including HIV prevention. Generally, expertise on behavior change has developed across a broad range of behavioral domains, and different researchers have different "pools of knowledge" about how to effectively design and implement theory-based interventions (e.g., different research experiences, analytic methods, and disciplinary training). Thus, the objective was to bring together an outstanding group of investigators from diverse disciplines and fields and provide them with a structured format and intellectually rich environment in which to generate the proposed integration.

This theoretical integration is likely to have a major impact on intervention design. The practical implications of the proposed theoretical integration are substantial. [1719]. For example, of the interventions meta-analyzed by Albarracín et al. [2], 51\% used theory as a formal basis for the intervention and $35 \%$ cited theories of behavior change. Thus, intervention researchers are eager to identify and use theory as a foundation for guiding the development, implementation, and evaluation of HIV prevention programs. Based on this meta-analytic evidence, we expect this supplement to be useful in future intervention design. In addition, the papers in this supplement provide investigators with a list of unanswered questions that can guide the allocation of time and effort and stimulate the development of an evidence base that can be used to further refine the proposed theoretical integration.

This theoretical integration is likely to stimulate future research syntheses. Various influential review papers have been inspired by or directly tested theoretical models. [3, 20] Thus, any expansion in the theories of health behavior change is likely to be the basis for future meta-analyses. This is important for maintaining a healthy, innovative cycle of theory production, primary research, synthesis of primary research and theory testing, then theory revision (see e.g.) [21].

This supplement. Theoretical models are best conveyed through the use of formal postulates as well as a graphical depiction of the relations among the key variables, mediators, and moderators. To make sense, the model must be presented in a logical order, with different sections addressing details of the explanations contained in the theory. After the Atlanta conference, members of each workgroup coauthored an article. Each article has a structured format to enhance comparability across articles, and the three core articles are followed by two papers, one coauthored by the editors and the other by the program officers from the National Institutes of Mental Health who participated. We have selected AIDS and Behavior to publish the special issue. AIDS and Behavior provides an update of HIV prevention research in the biobehavioral, psychological, and social sciences. The journal has gained an excellent reputation and is having considerable impact on HIV prevention research. We now turn to the papers and thank the authors for their work on the proposed theoretical integrations.

\section{References}

1. Fishbein M, Bandura A, Triandis HC, Kanfer FH, Becker MH, Middlestadt S. Factors influencing behavior, behavior change. Report prepared for the National Institute of Mental Health (NIMH). Bethesda, MD: NIMH; 1992.

2. Albarracín D, Gillette J, Earl A, Glasman LR, Durantini MR, Ho MH. A test of major assumptions about behavior change: a comprehensive look at HIV prevention interventions since the beginning of the epidemic. Psychol Bull. 2005;131:856-97.

3. Fisher JD, Fisher WA. Changing AIDS-risk behavior. Psychol Bull. 1992;111:455-74.

4. Barbanti Jr, O. Development interventions and conflict. Beyond intractability. In: Burgess $G$, Burgess $H$, editors. Conflict Research Consortium, University of Colorado, Boulder. Posted: August $2004<\mathrm{http} / / /$ www.beyondintractability.org/essay/develop ment_interventions/>.

5. Goodhand J. Violent conflict, poverty and chronic poverty. CPRC Working Paper. Chronic Poverty Research Center; 2001.

6. United Nations Development Program, Human Development Report. Millennium development goals: a compact among nations to end human poverty. New York: Oxford University Press; 2003.

7. Kerrigan D, Moreno L, Rosario S, Gomez B, Jerez H, Barrington C, Weiss E, Sweat M. Environmental-structural interventions to reduce HIV/STI risk among female sex workers in the Dominican Republic. Am J Public Health. 2004;96:120-5.

8. Latkin CA, Williams CT, Wang J, Curry AD. Neighborhood social disorder as a determinant of drug injection behaviors: a structural equation modeling approach. Health Psychol. 2005;24:96-100.

9. Stimson G. Drug injecting and HIV-infection. New directions for social-science research. Int J Addict. 1992;27:147-63.

10. Tawil O, Verster A, O'reilly KR. Enabling approaches for HIV/ AIDS Prevention: can we modify the environment and minimize the risk. AIDS. 1995;9:1299-306.

11. Varma D, Chandra PS, Thomas T, Carey MP. Intimate partner violence and sexual coercion among pregnant women in India: relationship with depression and post-traumatic stress disorder. J Affect Dis. 2007;102(1-3):227-35.

12. DiClemente RJ, Peterson JL, editors. AIDS prevention and mental health: handbook of HIV prevention. New York: Kluwer Academic/Plenum Publishers; 2000.

13. DiClemente RJ, Peterson JL. (edited). AIDS Prevention and Mental Health: Preventing AIDS: theories and methods of behavioral interventions. New York: Plenum Press (a division of Plenum Publishing Corp); 1994.

14. Ramirez-Valles J. The protective effects of community involvement for HIV risk behavior: a conceptual framework. Health Educ Res. 2002;17:389-403.

15. Cohen MS, Gay C, Kashuba A, Blower S, Paxton L. Narrative review: antiretroviral therapy to prevent the sexual transmission of HIV-1. Ann Internal Med 2007;146(8). 
16. Braun JF, Pozo MD. Understanding primary and secondary HIV prophylaxis-part 1: non-occupational post-exposure prophylaxis and antiretroviral pharmacokinetics in the male and female genital tracts. The PRN Notebook. Oct. 2006;11(2).

17. Rothman AJ. Toward a theory-based analysis of behavioral maintenance. Health Psychol. 2000;19:64-9.

18. Rothman AJ. Is there nothing more practical than a good theory?: Why Innovations and advances in health behavior change will arise if interventions are more theory-friendly. Int $\mathbf{J}$ Behav Nutri Physical Activity. 2004;1, 11
19. Suls J, Rothman AJ. Evolution of the psychosocial model: Implications for the future of health psychology. Health Psychol. 2004;23:119-25.

20. Albarracín D, Johnson BT, Fishbein M, Muellerleile P. Theories of reasoned action and planned behavior as models of condom use: a meta-analysis. Psychol Bull. 2001;127:142-61.

21. Eagly AH, Wood W. Using research syntheses to plan future research. In: Cooper H, Hedges LV, editors. The handbook of research synthesis. New York: Russell Sage; 1994. 